\title{
ClassRiskIndicator - Uma Ferramenta Móvel para Apoiar o Gerenciamento dos Riscos em Ambientes de Múltiplos Projetos com Base em Indicadores
}

\author{
Miguel Wanderley ${ }^{1}$, Júlio Menezes Júnior ${ }^{1}$, Cristine Gusmão ${ }^{1,2}$ \\ ${ }^{1}$ Grupo de Pesquisa SABER Tecnologias Educacionais e Sociais - Universidade Federal \\ de Pernambuco (UFPE) - Recife, PE - Brasil \\ ${ }^{2}$ Programa de Pós-graduação em Engenharia Biomédica - Centro de Tecnologia e \\ Geociências (CTG) - Universidade Federal de Pernambuco (UFPE) - Recife, PE - \\ Brasil \\ \{mdsw,jvmj\}@cin.ufpe.br, cristine.gusmao@pq.cnpq.br

\begin{abstract}
This paper presents the ClassRiskIndicator, a mobile indicator based tool aimed to assist the risk management of software development projects within a context of multiple projects environments. Its main goal is to help the project manager in the most important and basic tasks of the risk management process, in an objective, portable and simple way (relevant traits for an efficient risk management). This tool makes use of indicators that can characterize and summarize, in a unique value, the overall risk exposure level from a software development project.
\end{abstract}

Resumo. Este artigo apresenta o ClassRiskIndicator, uma ferramenta móvel baseada em indicadores que objetiva auxiliar o gerenciamento dos riscos de projetos de desenvolvimento de software em um ambiente de múltiplos projetos. O objetivo principal é ajudar o gerente de projetos nas tarefas mais importantes e fundamentais do processo de gestão de riscos, de forma objetiva, portátil e simples (traços relevantes para uma gestão eficiente dos riscos). Esta ferramenta faz uso de indicadores que podem caracterizar e resumir, em um único valor, o nível geral de exposição ao risco de um projeto de desenvolvimento de software.

\section{Introdução}

Projeto constitui em um esforço temporário empreendido para alcançar um objetivo específico, e.g., o desenvolvimento de um software. Projetos são executados por pessoas, geralmente apresentando limitações de recursos, sendo planejados, executados, controlados e documentados. Assim, um ambiente de projeto será um local, seja empresa ou qualquer organização, que realiza um ou mais projetos [PMI 2008].

Riscos são eventos que podem vir a afetar negativamente o desenvolvimento dos projetos ou o ambiente. Podem prejudicar principalmente fatores críticos como: orçamento, prazo ou recursos. Gerenciar riscos consiste basicamente em identificar, avaliar, planejar e controlar os eventos que ameaçam o ambiente do projeto, objetivando evitar ou diminuir os danos desses eventos caso eles ocorram de fato [Boehm 1991]. O gerenciamento, entretanto, não garante o sucesso dos projetos, porém eleva a probabilidade de um cumprimento mais efetivo, respeitando os prazos, dentro do 
orçamento e atendendo aos objetivos (requisitos) de cada projeto [Heldman 2005] [Almeida et al. 2012].

Nesse contexto, adotar indicadores representa ganhos nas atividades de gerenciamento e controle dos riscos do ambiente. Os benefícios relacionam-se com a aptidão de melhor controle e entendimento dos riscos e suas características (gravidade, origens, impacto etc.), assim como a avaliação mais eficiente do ambiente (maior precisão devido ao uso de valores reais coletados), melhor eficácia de predição e planejamento a respeito dos riscos e, por fim, uma capacidade de realizar melhorias nas atividades desenvolvidas durante o desenvolvimento dos projetos[Menezes Jr. 2013] [IOR 2010].

O presente trabalho foi organizado da seguinte forma: além desta seção introdutória, a Seção 2 apresenta uma visão geral do problema e a motivação para o desenvolvimento da ferramenta desenvolvida e um breve estudo sobre dispositivos e sistemas móveis. A Seção 3 descreve a ferramenta ClassRiskIndicator, com sua visão geral, arquitetura e funcionalidades. A Seção 4 apresenta um caso de uso real da ferramenta ilustrando a usabilidade e benefícios da mesma. E a Seção 5 apresenta um breve comparativo entre outras ferramentas similares existentes.

\section{Visão Geral e Motivação}

Adotar indicadores torna as atividades de controle e monitoramento dos riscos mais eficientes e corretas. São, assim, métricas que servem para medir a exposição ao risco do ambiente e dos projetos no tempo. Quando são adequados para a avaliação do ambiente, possibilitam identificar: i) tendências de riscos e eventos emergentes, ii) nível atual de exposição e iii) eventos que ocorreram no passado que podem ocorrer novamente. Para que um indicador possa ser entendido como adequado para o ambiente, este deve ter as seguintes características: i) ser relevante, ii) passível de medição, iii) preditivo, iv) fácil de monitorar, v) auditável e vi) comparável [IOR 2010].

Com o intuito de atribuir aos indicadores algumas dessas características, foi desenvolvida uma ferramenta móvel que confere as características de facilidade de monitorar (coletar, avaliar e interpretar, de forma fácil e eficiente) e auditável (igualmente fácil de ser acessado, examinado e comunicado). Além de operações automáticas, como a geração de relatórios preditivos (capazes de apontar tendências de crescimento ou decrescimento dos valores), cálculo de métricas e comparação entre dados. Todas estas funcionalidades em um sistema simples e objetivo, operando em um dispositivo móvel com elevada portabilidade. Dotado, assim, de características ideais para uma condução eficiente do gerenciamento dos riscos do ambiente [Wroblewski 2011].

\subsection{Dispositivos e Sistemas Móveis}

Os sistemas e dispositivos móveis (tablets e smartphones) apresentam um elevado crescimento mundial, conquistando cada vez mais espaço no mercado. Desta forma, estes dispositivos estão se tornando muito mais presentes e comuns entre os usuários, tornando seus sistemas e programas mais intuitivos e compreensíveis para um elevado número de consumidores de tecnologia, muito embora, em geral, as capacidades operacionais de aparelhos móveis sejam mais limitadas se comparados com os computadores pessoais convencionais (e.g., tamanho menor de tela e capacidade de processamento inferior) [Wroblewski 2011]. 
Dentre as características mais expressivas dos dispositivos móveis, está o fato de que um aparelho desses não ocupa o mesmo espaço físico de um computador convencional, e nem precisa estar instalado em um local fixo, podendo ser portado pelo usuário, configurando uma mobilidade confortável e facilitando o manuseio desses dispositivos. O tamanho limitado da tela faz com que os aplicativos móveis sejam mais objetivos, com funcionalidades e opções mais simples e uma interface mais limpa. [Wroblewski 2011].

\section{ClassRiskIndicator}

ClassRiskIndicator é uma ferramenta móvel para auxiliar o gerenciamento dos riscos em ambientes de múltiplos projetos de desenvolvimento de software. O sistema utiliza indicadores compostos por dados básicos para o gerenciamento dos riscos: probabilidade e impacto dos riscos identificados. Além das respostas de um questionário com perguntas referentes ao ambiente, relacionadas a fatores de risco mais relevantes.

A ferramenta elabora relatórios baseados em indicadores, os quais representam o nível de exposição ao risco de cada projeto desenvolvido no ambiente, ao longo do ciclo de vida dos projetos. Como entre as coletas algumas ações podem ser tomadas (planejamento dos riscos), a eficiência das ações tomadas pode ser avaliada de forma mais precisa. E um acompanhamento da quantidade de exposição aos riscos dos projetos pode ser realizado com base nesses relatórios, que além de apresentar o atual nível de exposição ao risco dos projetos (e o histórico desses valores), também podem apresentar tendências de crescimento ou decrescimento do valor dos indicadores, caracterizando uma expressiva ajuda para a tomada de decisões no ambiente.

A ferramenta utiliza inicialmente o indicador Ponto de Riscos [Oliveira 2006]. Este indicador é definido da seguinte maneira:

$$
P R=P R N A * \text { FCP }
$$

Onde PR é Pontos de Risco, PRNA é Pontos de Riscos Não Ajustados e FCP é o Fator Caracterizador do Projeto. O PRNA é uma adaptação do cálculo de Pontos de Casos de Uso Não Ajustados, enquanto que FCP é baseado em modelo de caracterização de projetos apresentado no trabalho de Oliveira (2006). Segundo o autor, o objetivo deste indicador é avaliar a complexidade de projetos de software com base na quantidade de riscos identificados.

Também foram feitas algumas modificações na forma de calcular o Ponto de Risco, essas modificações geraram novos indicadores alternativos que foram incorporados ao sistema. São os indicadores: i)Ponto de Risco Puro - sem a multiplicação do FCP e ii)Ponto de Risco Potência de 2 - com os fatores de multiplicação incrementados nas potências de $2(2,4,8,16$ e 32$)$. O primeiro busca encontrar valores de acordo com a simples multiplicação da probabilidade e do impacto (exposição ao risco). O segundo busca ser mais sensível em situações de alto risco, gerando valores mais expressivos para elevados níveis de exposição ao risco.

\subsection{Desenvolvimento}

O desenvolvimento foi feito seguindo as principais etapas de um desenvolvimento de software, com as fases de levantamento dos requisitos (que elucidaram as funcionalidades descritas mais a diante, com base no trabalho "Key Risk Indicators" 
[IOR 2010]). Fase de planejamento do projeto, modelagem, implementação e testes do sistema. Foi desenvolvido um protótipo para testes e aplicação em projetos reais.

\subsection{Arquitetura}

O sistema foi desenvolvido para a plataforma Android (versão 3.2, ou mais recente), escrito em Java (Eclipse IDE) e utilizando banco de dados SQLite. Segue a arquitetura MVC, onde um controlador processa os dado de entrada e exibe resultados em uma interface de usuário, além de existir um modelo para os dados operados no sistema, neste caso os indicadores e as informações necessárias para a composição deste. A visão geral da arquitetura da ferramenta é apresentada na Figura 1. Trata-se de um dispositivo móvel, com um armazenamento em banco de dados local, onde um usuário fornece dados que são armazenados e calculados, gerando relatórios os quais poderão ser lidos por outros interessados e estarão disponíveis para acesso.

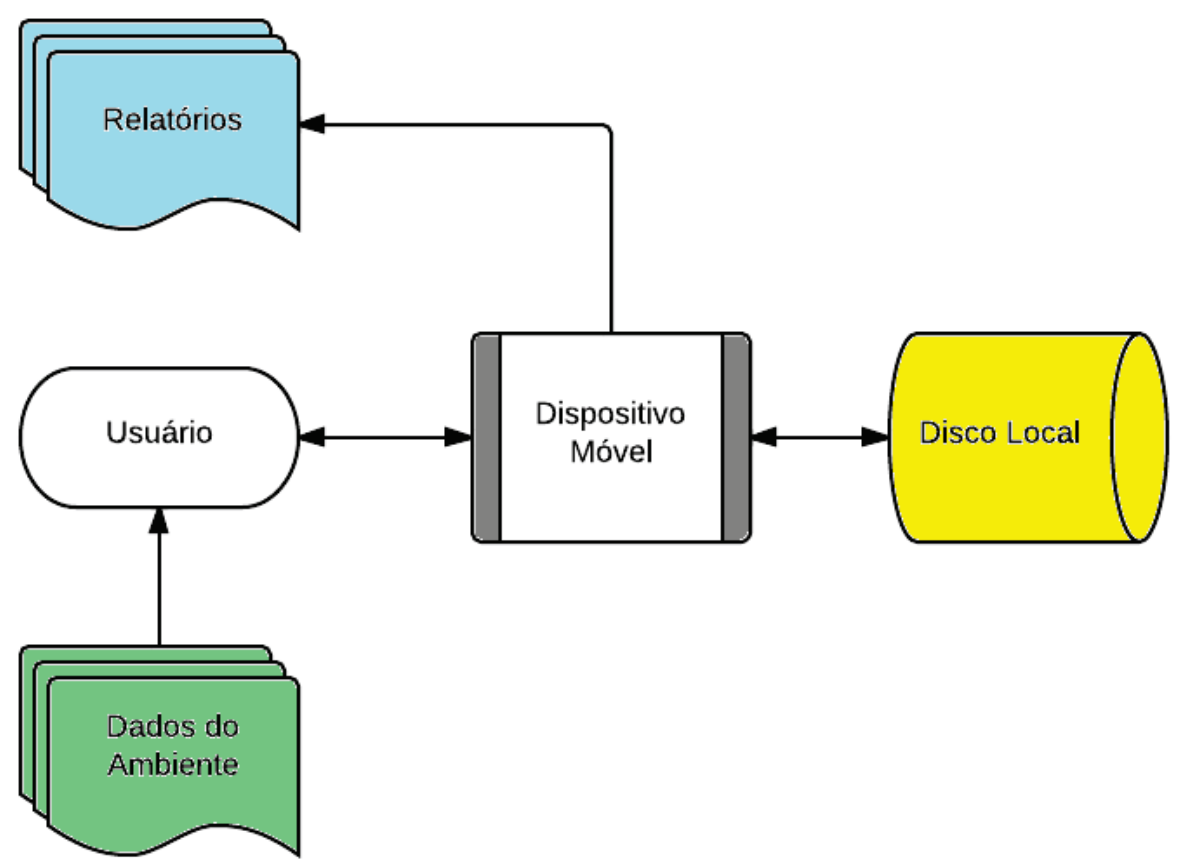

Figura 1: Arquitetura geral do sistema

Do ponto de vista lógico, a ferramenta se apresenta conforme a Figura 2. O sistema móvel (dispositivo móvel) apresenta uma Interface de Usuário, para a interação entre o usuário e o sistema (fornecer dados ao sistema, manutenção dos dados e acessar relatórios). Também apresenta uma unidade para Processamento de Dados do Indicador, para que o valor do indicador seja calculado com base nos dados coletados (Dados de Entrada). Com os indicadores calculados (Valor do Indicador) e outros dados de entrada, os relatórios são gerados na unidade Gerador de Relatórios. 


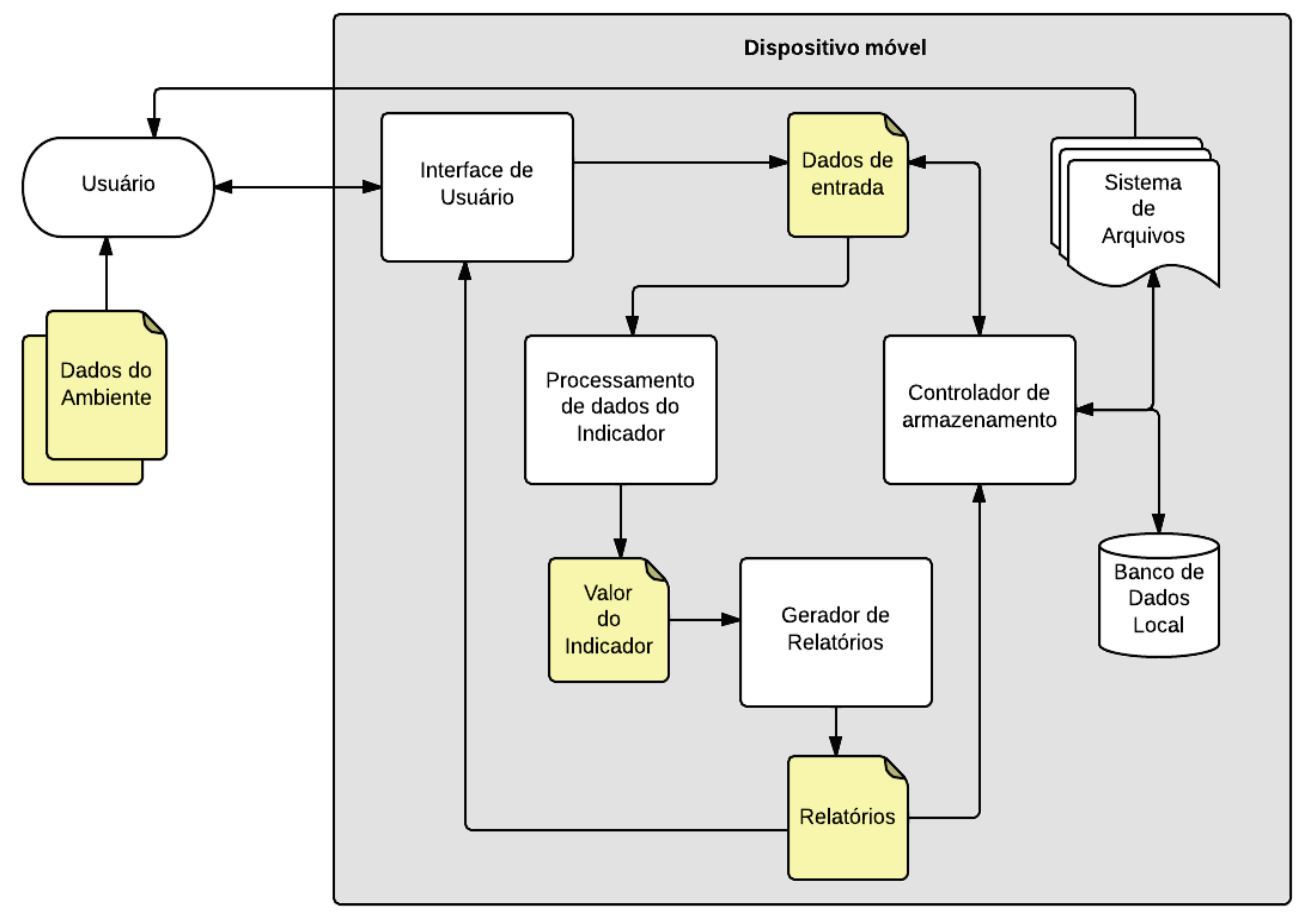

Figura 2: Esquema lógico da ferramenta ClassRiskIndicator

Uma vez gerados, os relatórios são apresentados para o usuário e enviados para a Interface de Usuário, e são enviados para a unidade Controlador de Armazenamento, onde são arquivados e armazenados no Banco de Dados Local e escritos em forma de arquivos no Sistema de Arquivos (tornando-se disponível para acessos e cópias), da mesma fora como é feito com os Dados de entrada, que são imediatamente armazenados (em forma de arquivos de $\log$ - arquivos texto e CSV) para diminuir a chance de perda desses dados.

\subsection{Funcionalidades}

A ferramenta ClassRiskIndicator busca auxiliar as principais atividades do gerenciamento dos riscos, ou seja, as atividades de identificação, avaliação, planejamento e controle dos riscos. A Figura 3 exemplifica a interface mais objetiva da ferramenta, com o objetivo de tornar a usabilidade do sistema mais eficiente.

As funcionalidades buscam atender os requisitos definidos durante a modelagem do sistema. Esses requisitos objetivam atender o maior quantidade de funcionalidades e características esperadas para indicadores de riscos, conforme descritas no trabalho do Institute of Operational Risk - IOR (2010). 


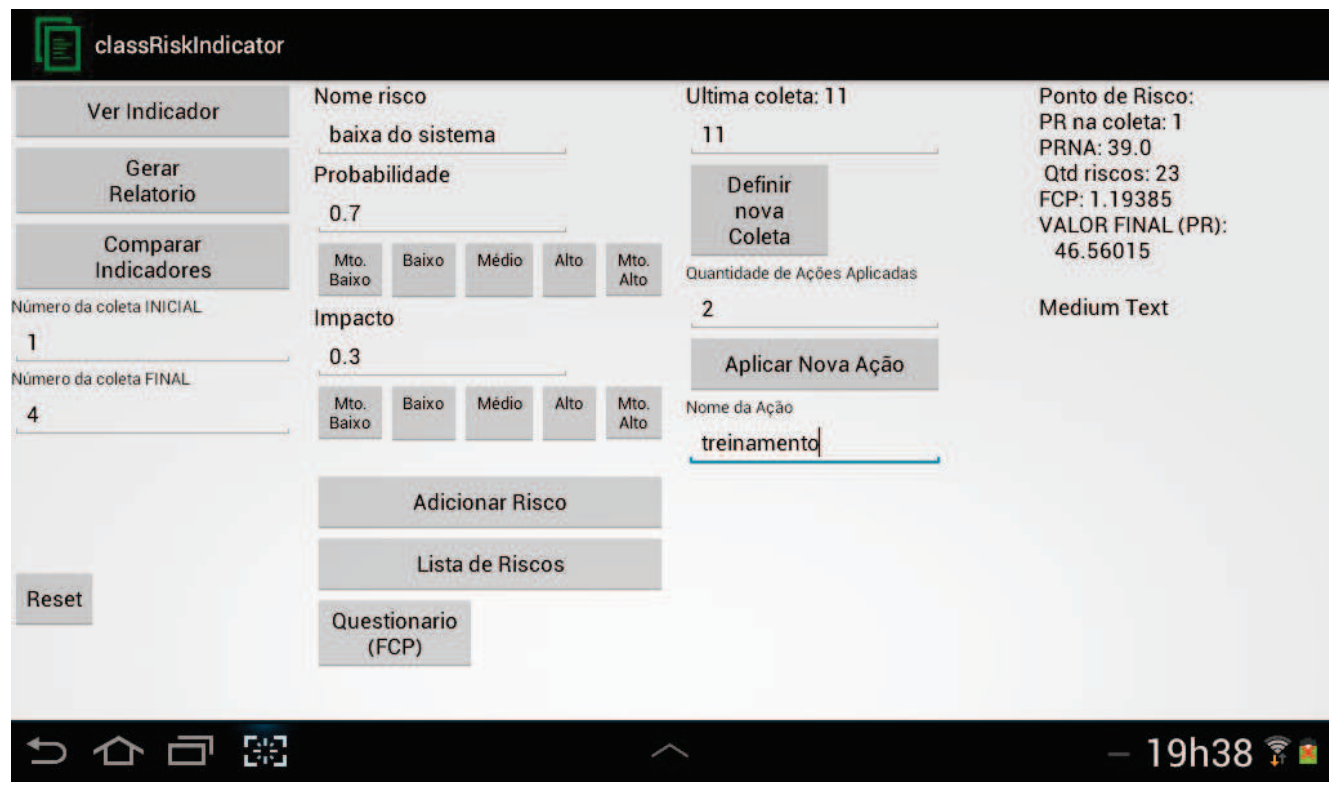

Figura 3: Exemplo da interface do sistema

Gerenciar Dados dos Riscos: Adicionar Valores de Coletas: Nome do risco, Probabilidade do Risco (escala de 1 até 5), Impacto do Risco (escala de 1 até 5).

Gerenciar Dados Coletados: Permite que coletas anteriores sejam visualizadas, modificadas e removidas.

Gerenciar Ações Planejadas: Permite que ações possam ser adicionadas ao sistema, tendo sua data de aplicação armazenada. Essas ações serão apresentadas nos relatórios posteriores, possibilitando a avaliação de suas eficácias.

Aplicar Questionários Sobre os Projetos: Realizar Perguntas Sobre o Projeto, necessário para o valor "Fator Caracterizador do Projeto" (FCP) para composição dos indicadores. Responder às Perguntas (escala de 1 até 5). Armazenar respostas. Modificar respostas coletadas.

Calcular Valores dos Indicadores: Compor os valores dos indicadores com base nos dados coletados referentes a cada coleta de dados.

Gerar Relatórios: Compor Dados das Coletas e dos Indicadores em Único Relatório. Compor Histórico das Coletas e Dados Coletados. Compor Relatório Relacionado Histórico dos Indicadores com Ações Tomadas. Gerar Relatórios em Formatos Diferentes (texto, tabelas e gráficos).

\section{Aplicação do Sistema}

A ferramenta foi aplicada experimentalmente em projetos de desenvolvimento de sistemas para Educação a Distância da UFPE. Para a execução deste estudo, foi aplicado o processo GARA - Gestão Ágil de Riscos de Ambiente, apoiada por atividades de identificação e análise de riscos. O processo GARA está descrito no trabalho "Definição de um Processo Ágil de Gestão de Riscos em Ambientes de Múltiplos Projetos" [RIBEIRO e GUSMÃO 2008]. Desta forma, o trabalho apresenta a integração entre indicador, processo e ferramenta. 


\subsection{Exemplo de Uso do Sistema}

Nesta seção será apresentado um exemplo de fluxo de uso do sistema, ilustrando a aplicação do mesmo em atividades de gerenciamento dos riscos em projetos.

Primeiramente deve-se pressionar "Definir Nova Coleta", assim, uma nova coleta de valores será automaticamente iniciada. $\mathrm{O}$ usuário irá identificar o projeto ao qual os valores estão sendo adicionados. Feito isso, os novos dados podem ser coletados, na mesma tela inicial, exemplificada na Figura 3.

Durante uma reunião de identificação de riscos, com o gerente de riscos e líderes das equipes presentes, os ricos vão sendo identificados e adicionados ao sistema. Para adicionar um risco deve-se adicionar o nome do risco no campo adequado e definir um valor para probabilidade e impacto nos respectivos campos (note que esses valorem apresentam teclas de atalho de acordo com uma escala de 5 níveis: "Muito Baixo", "Baixo", "Médio", "Alto" e "Muito Alto" que representam, respectivamente, os valores $0.1,0.3,0.5,0.7$ e 0.9 ). Preenchidos os campos, basta pressionar "Adicionar Risco" para que o mesmo seja arquivado no sistema.

Uma vez que coletas já foram realizadas, riscos de outras coletas (de outros ou do mesmo projeto) podem ainda ser observados na coleta atual, principalmente os riscos relativos ao ambiente. Sendo assim, a lista dos riscos pode ser acessada pressionando "Lista de Riscos" e então eles podem ser adicionados à coleta atual, apenas adicionando os valores de probabilidade e impacto e pressionando "Adicionar Risco". A Figura 4 exemplifica a interface desta funcionalidade, que se apresentou como uma boa alternativa durante uma reunião de brainstorming, agilizando o processo de identificação dos riscos.

\begin{tabular}{|c|c|c|c|c|c|}
\hline mud. ver. moodle & \multicolumn{5}{|c|}{$\begin{array}{l}\text { Risco: } \\
\text { perda de equipamento }\end{array}$} \\
\hline baixo conhecimento no moodle & \multicolumn{5}{|c|}{$\begin{array}{l}\text { Adicionar } \\
\text { Risco }\end{array}$} \\
\hline dificuldade de reuso moodle & \multicolumn{5}{|c|}{ Probabilidade } \\
\hline falha de comunicação da equipe & \multicolumn{5}{|l|}{0.5} \\
\hline perda de equipamento & $\begin{array}{l}\text { Mto. } \\
\text { Baixo }\end{array}$ & Baixo & Médio & Alto & $\begin{array}{l}\text { Mto. } \\
\text { Alto }\end{array}$ \\
\hline perda de prazo & \multicolumn{5}{|l|}{ Impacto } \\
\hline baixo nivel de gerenciamento & \multicolumn{5}{|l|}{0.7} \\
\hline falta/dia pessoa & $\begin{array}{l}\text { Mto. } \\
\text { Baixo }\end{array}$ & Baixo & Médio & Alto & $\begin{array}{l}\text { Mto. } \\
\text { Alto }\end{array}$ \\
\hline falta/dia Rodrigo & & & & & \\
\hline baixa capacidade do novo membr & & & & & \\
\hline adaptação do novo membro & & & & & \\
\hline
\end{tabular}

Figura 4: Interface da Lista de Riscos

Ao término da identificação dos riscos e adição dos valores de probabilidade e impacto dos mesmos, um questionário sobre o projeto deve ser respondido para a composição dos valores dos indicadores. Para iniciar o mesmo basta pressionar 
"Questionário (FCP)". A Figura 5 apresenta um exemplo da interface de aplicação do questionário, que também é respondido em uma escala de 5 níveis.

\begin{tabular}{|c|}
\hline Muito Pequena ( 1 a 6 pessoas) \\
\hline Pequena ( 7 a 20 pessoas) \\
\hline Média ( 21 a 50 pessoas) \\
\hline Grande ( 51 a 100 pessoas) \\
\hline Muito Grande (mais de 100 pessoas) \\
\hline
\end{tabular}

Figura 5: Interface do Questionário

Após esses passos de coleta dos dados referentes aos riscos, todos os valores já estão armazenados no Banco de Dados Local do sistema e em arquivos de log gerados, evitando que os valores sejam perdidos por eventuais falhas e permitindo que estes sejam arquivados em outros volumes à escolha do usuário. Neste momento, os valores dos indicadores também já foram devidamente calculados e armazenados no Banco de Dados Local e no Disco Local. Para acessar os resultados dos indicadores basta pressionar "Gerar Relatórios", então arquivos de relatórios serão gerados e armazenados no Disco Local. Os relatórios e os arquivos de $\log$ estarão presentes no Disco Local em formatos de arquivos de texto e no formato CSV, o qual pode ser visualizado na maioria dos leitores de planilhas.

Quando é solicitada a apresentação dos relatórios ("Gerar Relatórios") o sistema exibe um gráfico com os valores dos indicadores(eixo vertical) ao longo das coletas (eixo horizontal) de um projeto. A Figura 6 apresenta um exemplo deste gráfico. 


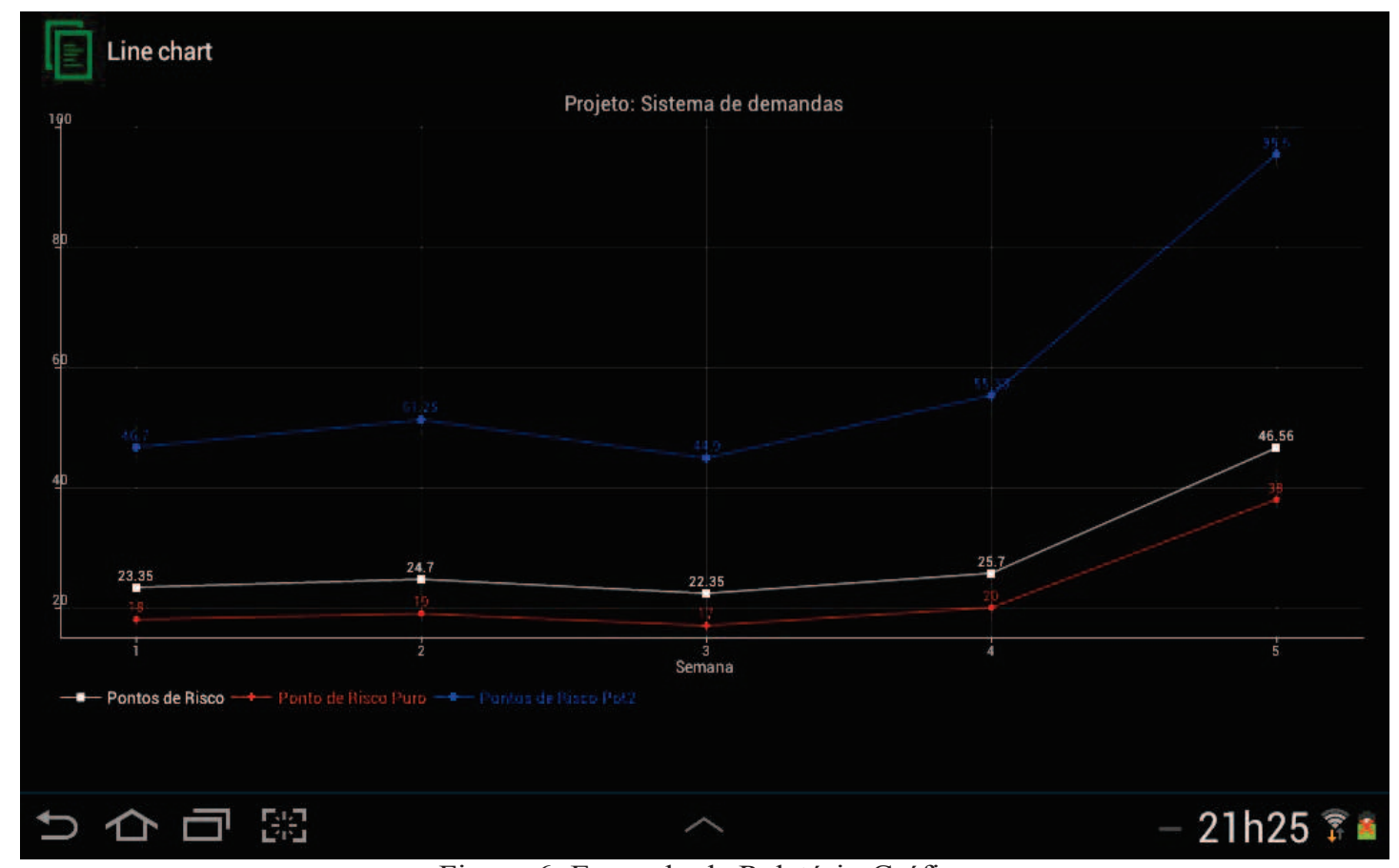

Figura 6: Exemplo de Relatório Gráfico

Também é possível visualizar a eficiência das ações aplicadas em um gráfico que apresenta o valor de um indicador e as ações aplicadas no intervalo entre as coletas. A Figura 7 ilustra esse gráfico. Vale lembrar que os indicadores revelam o grau de risco dos projetos e que os riscos de um projeto tende a crescer à medida que o mesmo avança no seu ciclo de vida [Boehm 1991].

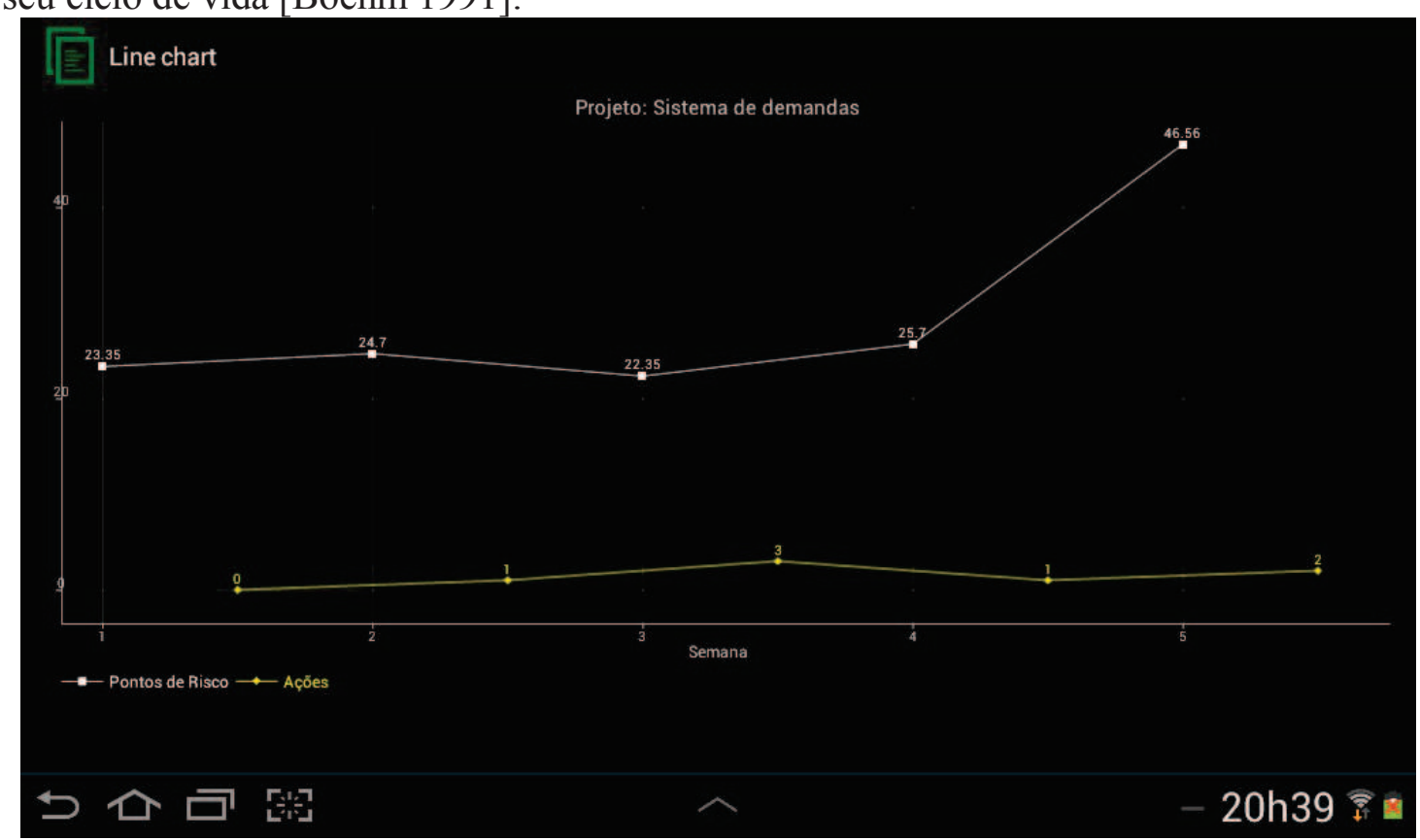

Figura 7: Exemplo de comparação Ações e Pontos de Risco 


\subsection{Resultados da Aplicação do Sistema}

As figuras apresentadas anteriormente foram aproveitadas do uso do sistema nos projetos elaborados no grupo de pesquisa SABER - Tecnologias Educacionais e Sociais da UFPE, que é um ambiente de múltiplos projetos de desenvolvimento de software web e mobile voltados para educação e gestão acadêmica de cursos.

As informações geradas no sistema, bem como os resultados dos indicadores, possibilitaram um entendimento mais preciso dos riscos dos projetos e do ambiente. Foi possível identificar quais os riscos compartilhados entre os projetos, i.e., os riscos do ambiente. Isso permitiu a elaboração de planos de ações mais contundentes para evitar ou mitigar tais riscos. Além de planos e ações para lidar com os riscos dos projetos específicos.

A eficiência das ações e estratégias adotadas nos projetos, assim como o impacto delas, também ficaram mais fáceis de serem monitoradas pelo gerente de riscos e posteriormente serem comunicadas aos líderes das equipes.

A priorização dos riscos foi feita de forma mais eficiente e automática a partir dos relatórios gerados. As planilhas também puderam ser utilizadas para elaboração de relatórios sobre os projetos.

\section{Ferramentas Relacionadas}

A Tabela 1 um apresenta um breve estudo comparativo de ferramentas de gerenciamento de riscos relacionadas: Risk Radar ${ }^{1}$, Risk Guide ${ }^{2}$, Risk Free ${ }^{3}$, XeroRisk $^{4}$, @RISK $6{ }^{5}$ e mPRIME Tool ${ }^{6}$.

Tabela 1: Comparativo entre ferramentas similares

\begin{tabular}{|l|l|l|l|}
\hline & $\begin{array}{l}\text { Projetos de } \\
\text { Software }\end{array}$ & Plataforma & Relatórios \\
\hline Risk Radar & Não (Genérico) & Desktop/Web & Diversos \\
\hline Risk Guide & $\begin{array}{l}\text { Sim } \\
\text { (Específico) }\end{array}$ & Desktop & Poucos \\
\hline Risk Free & $\begin{array}{l}\text { Sim } \\
\text { (Específico) }\end{array}$ & Desktop & Poucos \\
\hline XeroRisk & Não (Genérico) & Web & Poucos \\
\hline @RISK 6 & Não (Genérico) & $\begin{array}{l}\text { Desktop + Microsoft } \\
\text { Excel }\end{array}$ & Diversos \\
\hline mPRIME Tool & $\begin{array}{l}\text { Sim } \\
\text { (Específico) }\end{array}$ & Desktop & Diversos \\
\hline ClassRiskIndicator & $\begin{array}{l}\text { Sim } \\
\text { (Específico) }\end{array}$ & Mobile (Android) & Diversos \\
\hline
\end{tabular}

De modo geral, as principais ferramentas presentes no mercado são ferramentas não específicas para projetos de software e dependentes de um computador, existindo poucas alternativas móveis. Além de serem geralmente pagas.

\footnotetext{
${ }^{1}$ Disponível em http://www.mitre.org/work/sepo/toolkits/risk/ToolsTechniques/RiskRadar.html

${ }^{2}$ Disponível em http://iag.pg.gda.pl/RiskGuide/demo.html

${ }^{3}$ Disponível em http://www.inf.pucrs.br/ rafael/RiskFree/

${ }^{4}$ Disponível em: http://www.xerorisk.com

${ }^{5}$ Disponível em http://www.palisade.com/risk/

${ }^{6}$ Disponível em: http://www.cin.ufpe.br/ suppera/mprime.php
} 


\section{Considerações Finais}

Este trabalho apresentou um sistema móvel que utiliza indicadores para facilitar o gerenciamento dos riscos em ambientes de múltiplos projetos de desenvolvimento de software. O sistema torna mais eficiente o processo de coleta de dados, cálculo dos indicadores e geração de relatórios específicos para a avaliação dos riscos do ambiente e projetos como um todo. A característica móvel da ferramenta implica nas vantagens de possibilidade de descentralização e mobilidade do processo de coleta e manipulação mais eficiente das informações. Além de apresentar uma interface mais objetiva, tornando a usabilidade da ferramenta mais intuitiva.

A ferramenta apresenta-se em fase de melhorias, sendo aplicada em projetos reais da Universidade Federal de Pernambuco, juntamente com um processo ágil de gestão de riscos: GARA [Ribeiro e Gusmão 2008]. A continuidade deste trabalho também pode ser feita com o desenho de uma ferramenta mais genérica, que suporte o uso de diferentes indicadores. Além da adição de mais funcionalidades e refino das atuais, sem comprometer a usabilidade do sistema.

A ferramenta ClassRiskIndicator pode ser encontrada no endereço: http://cin.ufpe.br/ mdsw/classriskindicator.

\section{Agradecimentos}

Os autores agradecem à FACEPE e ao $\mathrm{CNPq}$ pelo apoio necessário à execução deste trabalho.

\section{Referências}

Almeida, K., Gusmão, C., Menezes Jr., J. e Cruz Neto, G. (2012) "Estrutura Analítica de Riscos para Ambientes de Múltiplos Projetos de Software", CONTECSI, International Conference on Information Systems and Technology Management.

Boehm, B. W. (1991) "Software Risk Management: Principles and Practices”.

Heldman, K. (2005) "Project Manager's Spotlight on Risk Management”, Harbor Light Press, Londres.

IOR, Institute of Operational Risk. (2010) "Key Risk Indicators", Operational Risk Sound Practice Guidance.

Menezes Jr., J., Gusmão, C., Moura, H. (2013) "Defining Indicators for Risk Assessment in Software Development Projects". CLEI Electronic Journal, V. 16, 1, abril/2013.

Oliveira, S. L. S.(2006) "Análise e Definição de Métricas para o Processo de Gerência de Riscos para Projetos de Software". Trabalho de graduação, Centro de Informática/UFPE.

PMI, Project Management Institute. (2008) "Project Management Body of Knowledge" (PMBOK). 4a ed.

Ribeiro, L., Gusmão, C. (2008) "Definição de um Processo Ágil de Gestão de Riscos em Ambientes de Múltiplos Projetos". Hífen, Uruguaiana, v. 32, 62, p. 67-74.

Wroblewski, L. (2011) "Mobile First", A Book Apart, New York. 